\title{
Supplementary Materials for "A Hidden Spatial-temporal Markov Random Field Model for Network-based Analysis of Time Course Gene Expression Data"
}

\author{
Zhi Wei and Hongzhe $\mathrm{Li}^{*}$ \\ Department of Biostatistics and Epidemiology \\ University of Pennsylvania School of Medicine, Philadelphia, PA 19104, USA
}

*Address correspondence to:

Hongzhe Li

Department of Biostatistics and Epidemiology University of Pennsylvania School of Medicine

Philadelphia, PA 19104, USA.

Tel: (215) 573-5038

Email: hongzhe@mail.med.upenn.edu 
Table S1 shows detailed Table 1 in the paper, including the standard errors of the estimates of the sensitivities, specificities and FDRs.

Table S2 shows detailed Table 2 in the paper, including the standard errors of the estimates of the sensitivities, specificities and FDRs.

Figure S1 shows the average gene expression profiles (+/-1SE) at the $0 \mathrm{~h}, 2 \mathrm{~h}$ and $4 \mathrm{~h}$ for the 35 DE genes that were identified by the hstMRF model but missed by the HMM model.

Figure S2 shows the average gene expression profiles (+/-1SE) at $0 \mathrm{~h}, 2 \mathrm{~h}$ and $24 \mathrm{~h}$ for the $56 \mathrm{DE}$ genes that were identified by the hstMRF model but missed by the HMM model when only the data at the $0 \mathrm{~h}, 2 \mathrm{~h}$ and $24 \mathrm{~h}$ were used. 


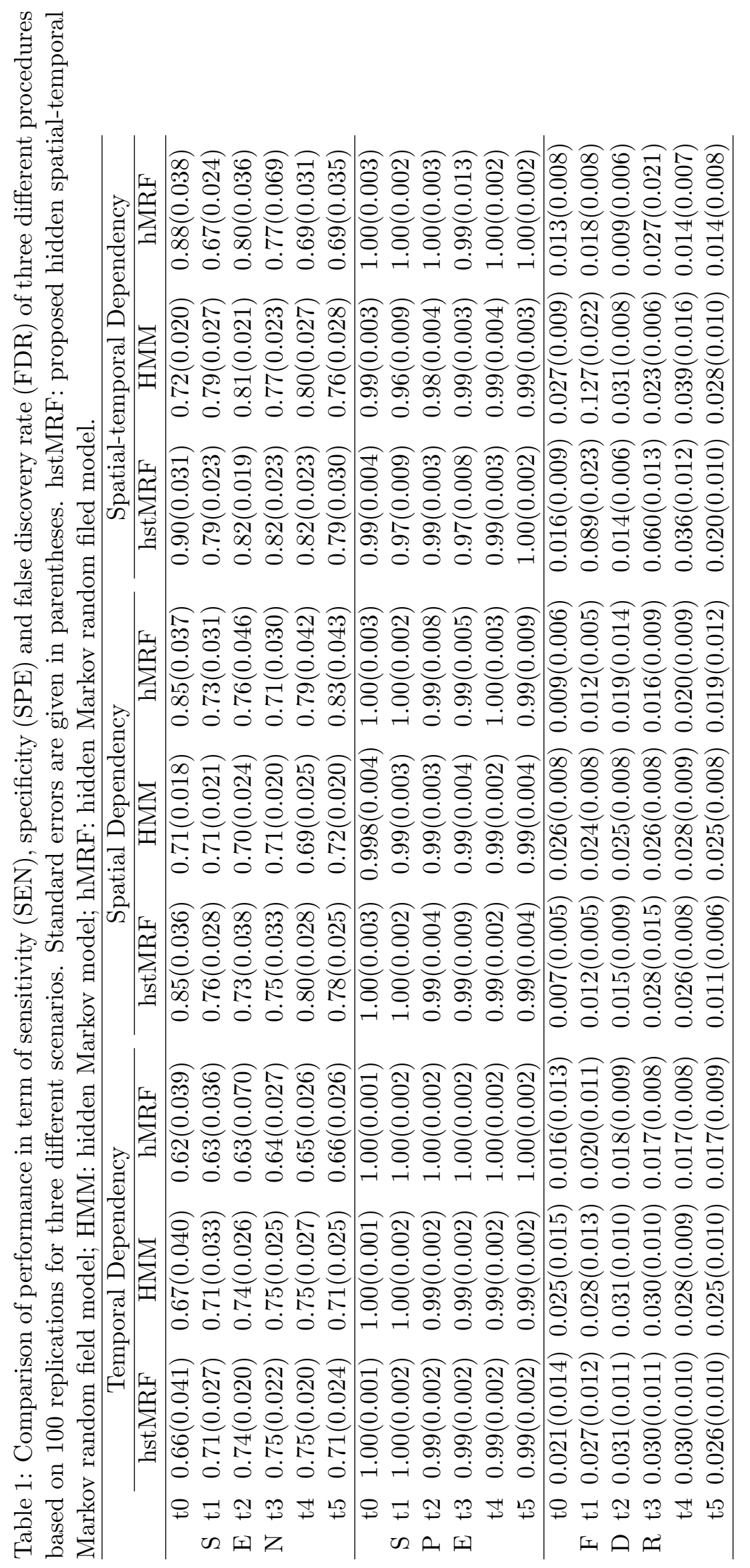




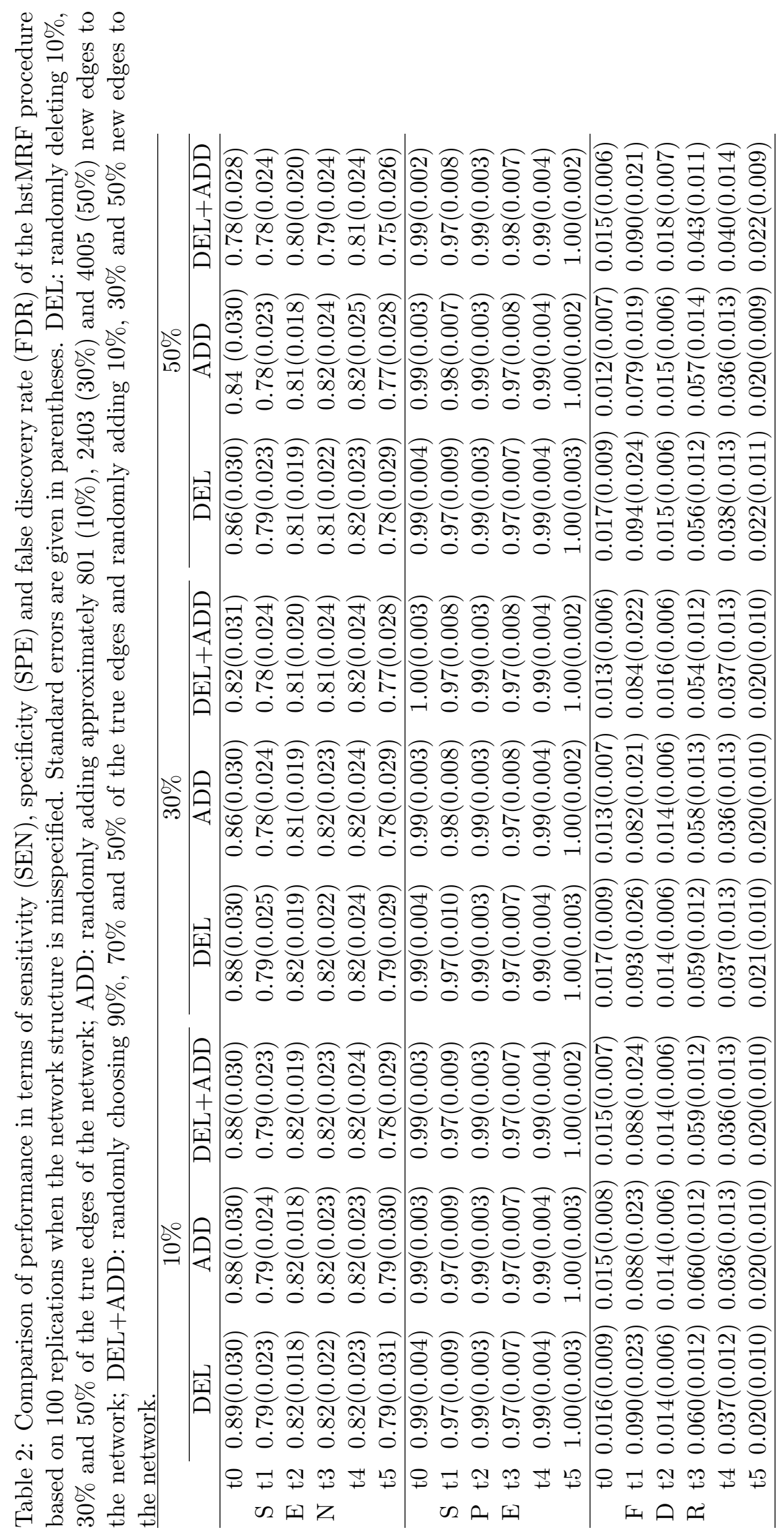



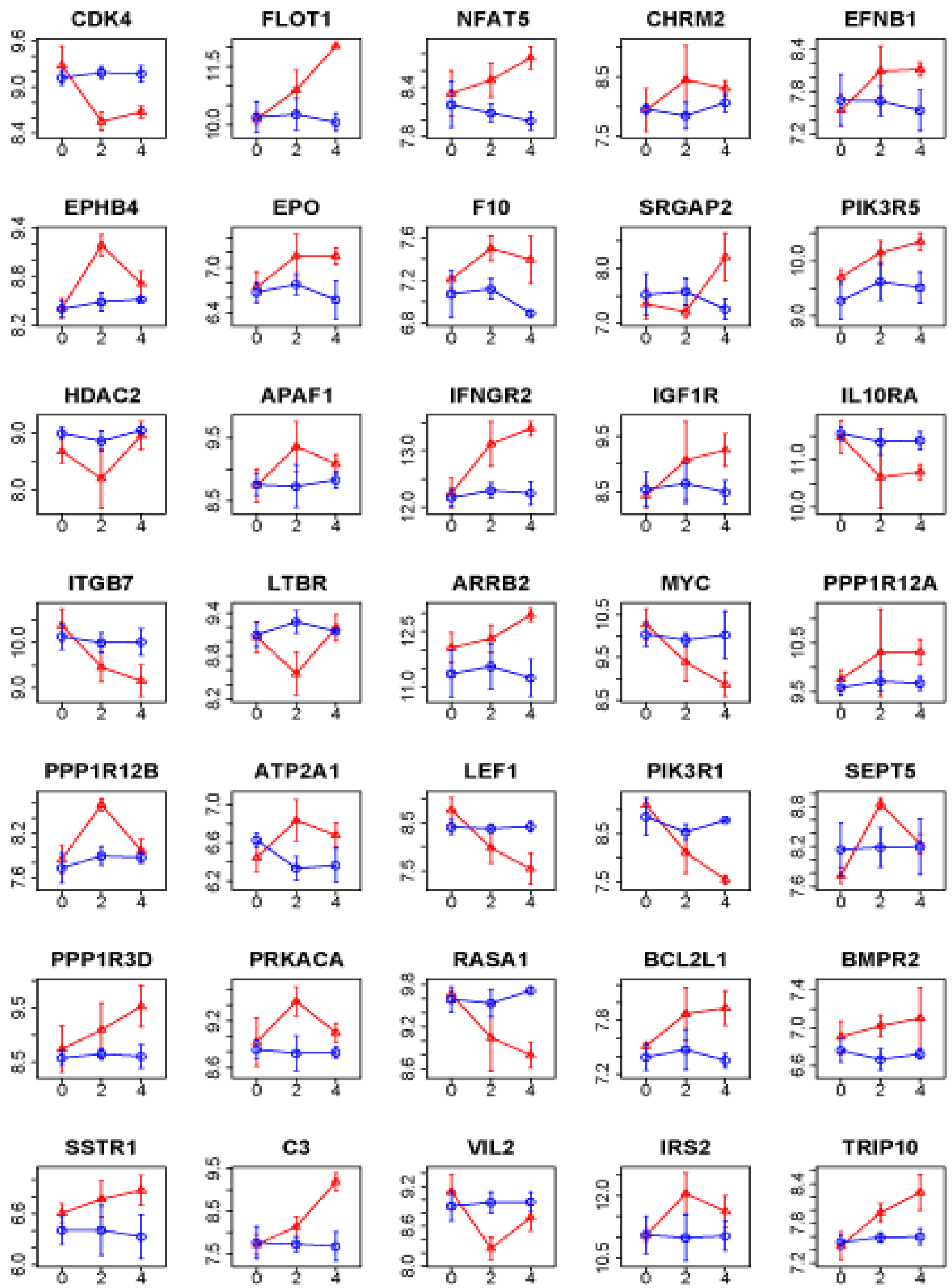

Figure 1: Average gene expression measures from the RMA procedure (in $\log _{2}$ scale) $(+/-1 \mathrm{SE})$ (y-axis) at the $0 \mathrm{~h}, 2 \mathrm{~h}$ and $4 \mathrm{~h}$ (x-axis) for the $35 \mathrm{DE}$ genes that were identified by the hstMRF model but missed by the HMM model. $\Delta$ : group receiving the endotoxin administration; $o$ : control group. 

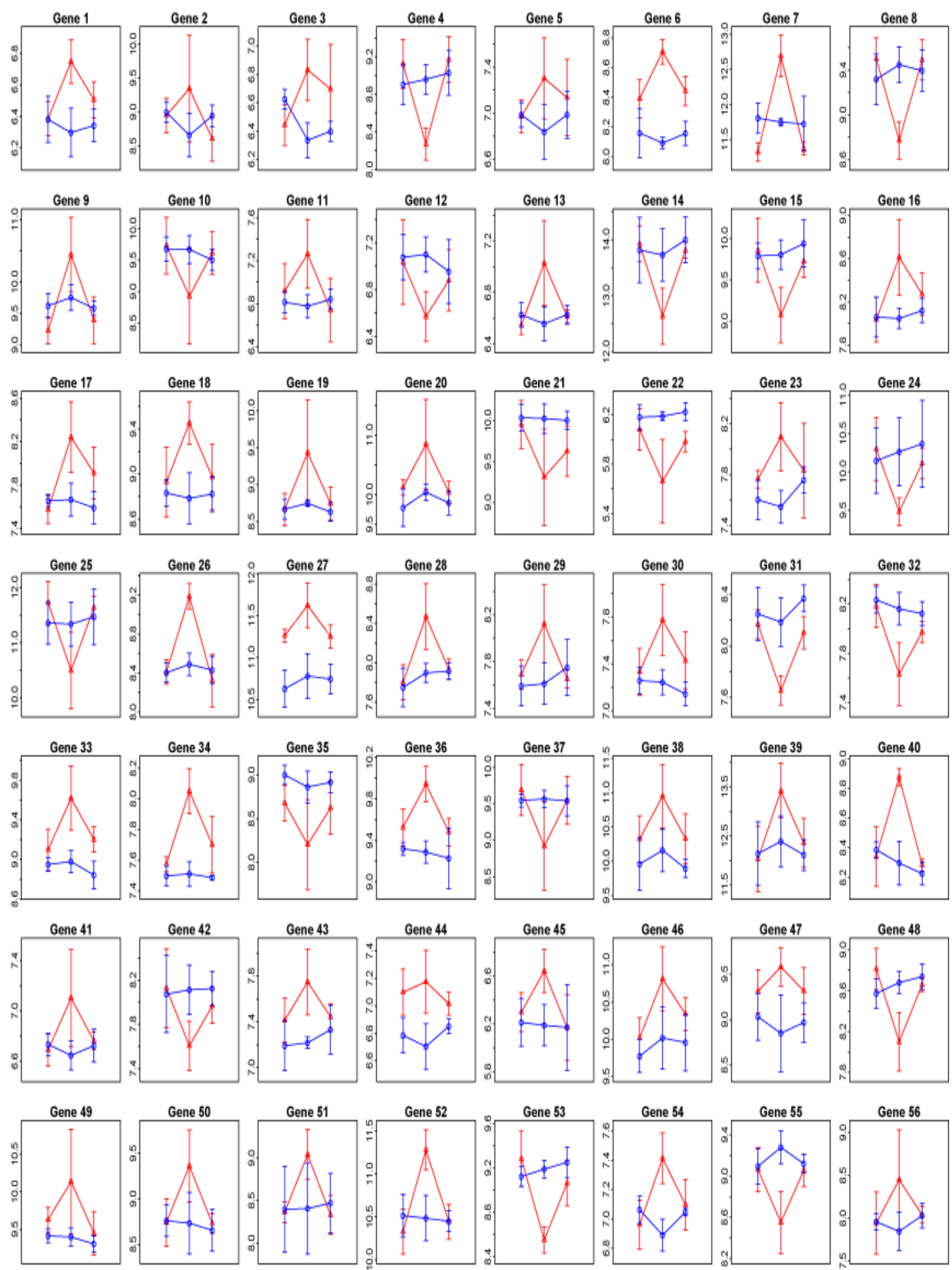

Figure 2: Average gene expression measures from the RMA procedure (in $\log _{2}$ scale) $(+/-1 \mathrm{SE}$ ) (y-axis) at $0 \mathrm{~h}, 2 \mathrm{~h}$ and $24 \mathrm{~h}$ (x-axis) for the $56 \mathrm{DE}$ genes that were identified by the hstMRF model but missed by the HMM model. $\Delta$ : group receiving the endotoxin administration; $o$ : control group. 